\title{
Pragmatic View of Short-Baseline Neutrino Oscillations
}

\author{
C. Giunti \\ INFN, Sezione di Torino, Via P. Giuria 1, I-10125 Torino, Italy \\ M. Laveder \\ Dipartimento di Fisica e Astronomia "G. Galilei", Università di Padova, \\ and INFN, Sezione di Padova, Via F. Marzolo 8, I-35131 Padova, Italy \\ Y.F. Li \\ Institute of High Energy Physics, Chinese Academy of Sciences, Beijing 100049, China \\ H.W. Long \\ Department of Modern Physics, University of Science and Technology of China, Hefei, Anhui 230026, China
}

(Dated: Thursday 07/11/13, 01:30)

\begin{abstract}
We present the results of global analyses of short-baseline neutrino oscillation data in $3+1,3+2$ and $3+1+1$ neutrino mixing schemes. We show that the data do not allow us to abandon the simplest $3+1$ scheme in favor of the more complex $3+2$ and $3+1+1$ schemes. We present the allowed region in the $3+1$ parameter space, which is located at $\Delta m_{41}^{2}$ between 0.82 and $2.19 \mathrm{eV}^{2}$ at $3 \sigma$. The case of no oscillations is disfavored by about $6 \sigma$, which decreases dramatically to about $2 \sigma$ if the LSND data are not considered. Hence, new high-precision experiments are needed to check the LSND signal.
\end{abstract}

PACS numbers: 14.60.Pq, 14.60.Lm, 14.60.St

The possibility of short-baseline neutrino oscillations due to the existence of one or more sterile neutrinos at the $\mathrm{eV}$ scale is a hot topic in current neutrino physics (see [1-4]). Besides the intrinsic interest in determining the existence of new phenomena and particles, the existence and properties of sterile neutrinos and active-sterile mixing could shed light on the physics beyond the Standard Model (see [5, 6] ). The existence of light sterile neutrinos is also very important for astrophysics (see [7]) and cosmology (see [8, 9] ), and the recent first Planck results [10] have generated interesting studies on the implications of cosmological data for light sterile neutrinos [11 17$]$.

In this paper we extend the analysis of short-baseline electron neutrino and antineutrino disappearance data presented in Ref. [18] by taking into account also the more controversial indication of the LSND [19] experiment in favor of short-baseline $\bar{\nu}_{\mu} \rightarrow \bar{\nu}_{e}$ transitions and the recent ambiguous results of the MiniBooNE [20] experiment. We consider $3+1$ and $3+2$ extensions of the Standard Model in which there are, respectively, one or two sterile neutrinos at the $\mathrm{eV}$ scald 11 which generate short-baseline oscillations [18, 31 39]. In the $3+1$ scheme electron and muon neutrino and antineutrino appearance

1 Our approach does not exclude the possible existence of more sterile neutrinos as long as the effects of their mixing with the active neutrino is negligible for the analysis of current shortbaseline neutrino oscillation experiments. Heavier sterile neutrinos with masses at the $\mathrm{keV}$ scale have been considered as candidates for warm dark matter (see 21 24]). Very light sterile neutrinos with masses smaller than about $0.1 \mathrm{eV}$ can induce observable effects in solar neutrino experiments [25, 26] and in long-baseline reactor experiments $27-30]$. and disappearance in short-baseline experiments depend on:

A) One neutrino squared-mass difference, $\Delta m_{41}^{2}=$ $m_{4}^{2}-m_{1}^{2}$, where $m_{k}$ is the mass of the massive neutrino $\nu_{k}$ and $\Delta m_{21}^{2} \ll \Delta m_{31}^{2} \ll \Delta m_{41}^{2} \sim 1 \mathrm{eV}^{2}$ in order to accommodate the observed oscillations of solar, reactor, atmospheric and accelerator neutrinos in the standard framework of three-neutrino mixing (see [1, 40] ). The probability of $\stackrel{(-)}{\nu} \rightarrow \stackrel{(-)}{\nu}$ transitions has the two-neutrino-like form

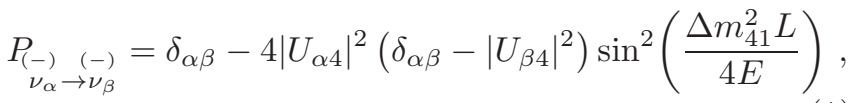

where $U$ is the mixing matrix, $L$ is the sourcedetector distance, and $E$ is the neutrino energy.

B) $\left|U_{e 4}\right|^{2}$ and $\left|U_{\mu 4}\right|^{2}$, which determine the amplitude $\sin ^{2} 2 \vartheta_{e \mu}=4\left|U_{e 4}\right|^{2}\left|U_{\mu 4}\right|^{2}$ of $\stackrel{(-)}{\nu_{\mu}} \rightarrow \stackrel{(-)}{\nu}$ transitions, the amplitude $\sin ^{2} 2 \vartheta_{e e}=4\left|U_{e 4}\right|^{2}\left(1-\left|U_{e 4}\right|^{2}\right)$ of $\stackrel{(-)}{\nu_{e}}$ disappearance, and the amplitude $\sin ^{2} 2 \vartheta_{\mu \mu}=$ $4\left|U_{\mu 4}\right|^{2}\left(1-\left|U_{\mu 4}\right|^{2}\right)$ of $\stackrel{(-)}{\nu_{\mu}}$ disappearance.

Since the oscillation probabilities of neutrinos and antineutrinos are related by a complex conjugation of the elements of the mixing matrix (see [40]), the probabilities of short-baseline $\nu_{\mu} \rightarrow \nu_{e}$ and $\bar{\nu}_{\mu} \rightarrow \bar{\nu}_{e}$ transitions are equal. Hence, the $3+1$ scheme cannot explain a possible CP-violating difference of $\nu_{\mu} \rightarrow \nu_{e}$ and $\bar{\nu}_{\mu} \rightarrow \bar{\nu}_{e}$ transitions in short-baseline experiments. In order to allow this possibility, one must consider a $3+2$ scheme, in which, there are four additional effective mixing parameters in short-baseline experiments: 
C) $\Delta m_{51}^{2}$, which is conventionally assumed $\geq \Delta m_{41}^{2}$.

D) $\left|U_{e 5}\right|^{2}$ and $\left|U_{\mu 5}\right|^{2}$.

E) $\eta=\arg \left[U_{e 4}^{*} U_{\mu 4} U_{e 5} U_{\mu 5}^{*}\right]$. Since this complex phase appears with different signs in $\nu_{\mu} \rightarrow \nu_{e}$ and $\bar{\nu}_{\mu} \rightarrow \bar{\nu}_{e}$ transitions, it can generate measurable $\mathrm{CP}$ violations.

In the analysis of short-baseline data, we consider the following three groups of experiments:

1. The $\stackrel{(-)}{\nu} \rightarrow \stackrel{(-)}{\nu}$ appearance data of the LSND 19], MiniBooNE [20], BNL-E776 [41], KARMEN 42], NOMAD [43], ICARUS [44] and OPERA [45] experiments.

2. The $\stackrel{(-)}{\nu_{e}}$ disappearance data described in Ref. [18], which take into account the reactor [46 48] and Gallium [49 53] anomalies.

3. The constraints on ${ }^{(-)} \nu_{\mu}$ disappearance obtained from the data of the CDHSW experiment [54], from the analysis [55] of the data of atmospheric neutrino oscillation experiment:2, from the analysis [33] of the MINOS neutral-current data [58] and from the analysis of the SciBooNE-MiniBooNE neutrino [59] and antineutrino [60] data.

With respect to the analysis presented in Ref. [38], we have added the new constraints on $\nu_{\mu} \rightarrow \nu_{e}$ transitions obtained in the ICARUS [44] and OPERA [45] experiments. Following Ref. 39], we also added the constraints on $\nu_{\mu} \rightarrow \nu_{e}$ and $\bar{\nu}_{\mu} \rightarrow \bar{\nu}_{e}$ appearance obtained in the old BNL-E776 [41] experiment, which give a small contribution at $\Delta m^{2}$ 's larger than about $2 \mathrm{eV}^{2}$, and the subleading effect of background disappearance in the analysis of MiniBooNE [20] data.

Table I shows the results of the $3+1$-LOW fit of all the data above, including the three low-energy bins of the MiniBooNE experiment whose excess with respect to the background is widely considered to be anomalous because it is at odds with neutrino oscillations [33, 34]. We have considered also a $3+1$-HIG fit of MiniBooNE data without the three anomalous low-energy bins. From Tab. I. one can see that in both cases the oscillation fit of the data is much better than the no-oscillation fit, which has a disastrous p-value and is excluded in both cases at about $6 \sigma$. Although the best-fit values of the oscillation parameters are similar in the $3+1$-LOW and $3+1$-HIG fits, the goodness-of-fit of the $3+1$-LOW case is significantly lower and the appearance-disappearance parameter goodness-of-fit is much lower. This result confirms the

2 We do not consider the IceCube data which could give a marginal contribution [56, 57], because the analysis is too complicated and subject to large uncertainties.

\begin{tabular}{|c|c|c|c|c|c|}
\hline & & LOW & HIG & noMB & noLSND \\
\hline No & $\chi^{2}$ & 339.2 & 308.0 & 283.2 & 286.7 \\
\hline \multirow[t]{2}{*}{ Osc. } & NDF & 259 & 253 & 221 & 255 \\
\hline & GoF & $0.06 \%$ & $1 \%$ & $0.3 \%$ & $8 \%$ \\
\hline $3+1$ & $\chi_{\min }^{2}$ & 291.7 & 261.8 & 236.1 & 278.4 \\
\hline \multirow[t]{15}{*}{ Osc. } & NDF & 256 & 250 & 218 & 252 \\
\hline & GoF & $6 \%$ & $29 \%$ & $19 \%$ & $12 \%$ \\
\hline & $\Delta m_{41}^{2}\left[\mathrm{eV}^{2}\right]$ & 1.6 & 1.6 & 1.6 & 1.7 \\
\hline & $\left|U_{e 4}\right|^{2}$ & 0.033 & 0.03 & 0.03 & 0.024 \\
\hline & $\left|U_{\mu 4}\right|^{2}$ & 0.012 & 0.013 & 0.014 & 0.0073 \\
\hline & $\sin ^{2} 2 \vartheta_{e \mu}$ & 0.0016 & 0.0015 & 0.0017 & 0.0007 \\
\hline & $\sin ^{2} 2 \vartheta_{e e}$ & 0.13 & 0.11 & 0.12 & 0.093 \\
\hline & $\sin ^{2} 2 \vartheta_{\mu \mu}$ & 0.048 & 0.049 & 0.054 & 0.03 \\
\hline & $\left(\chi_{\min }^{2}\right)_{\mathrm{APP}}$ & 99.3 & 77.0 & 50.9 & 91.8 \\
\hline & $\left(\chi_{\min }^{2}\right)_{D I S}$ & 180.1 & 180.1 & 180.1 & 180.1 \\
\hline & $\Delta \chi_{\mathrm{PG}}^{2}$ & 12.7 & 4.8 & 5.1 & 6.4 \\
\hline & $\mathrm{NDF}_{\mathrm{PG}}$ & 2 & 2 & 2 & 2 \\
\hline & $\mathrm{GoF}_{\mathrm{PG}}$ & $0.2 \%$ & $9 \%$ & $8 \%$ & $4 \%$ \\
\hline & $\mathrm{p}-\mathrm{val}_{\text {No Osc. }}$ & $3 \times 10^{-10}$ & $5 \times 10^{-10}$ & $3 \times 10^{-10}$ & $4 \times 10^{-2}$ \\
\hline & $n \sigma_{\text {No Osc. }}$ & $6.3 \sigma$ & $6.2 \sigma$ & $6.3 \sigma$ & $2.1 \sigma$ \\
\hline
\end{tabular}

TABLE I. Results of the fit of short-baseline data taking into account all MiniBooNE data (LOW), only the MiniBooNE data above $475 \mathrm{MeV}$ (HIG), without MiniBooNE data (noMB) and without LSND data (noLSND). The results of the fit without neutrino oscillations are given in the first three lines, whereas the other lines refer to the $3+1$ fit. We list the $\chi^{2}$, the number of degrees of freedom (NDF), the goodness-offit (GoF), the best-fit values of the $3+1$ oscillation parameters and the quantities relevant for the appearance-disappearance (APP-DIS) parameter goodness-of-fit (PG) 61]. In the last three lines we give the $\mathrm{p}$-value $\left(\mathrm{p}-\mathrm{val}_{\mathrm{No}}\right.$ Osc. $)$ and the corresponding number of excluding $\sigma$ 's $\left(n \sigma_{\text {No Osc. }}\right)$ of the nooscillation case.

fact that the MiniBooNE low-energy anomaly is incompatible with neutrino oscillations, because it would require a small value of $\Delta m_{41}^{2}$ and a large value of $\sin ^{2} 2 \vartheta_{e \mu}$ 33, 34], which are excluded by the data of other experiments. Indeed, one can see from Fig. 1 that the best-fit $3+1$-LOW averaged transition probability is far from fitting the three anomalous low-energy bins of MiniBooNE neutrino and antineutrino data. Therefore, we think that it is very likely that the MiniBooNE low-energy anomaly has an explanation which is different from neutrino oscillations 3 and the $3+1-\mathrm{HIG}$ fit is more reliable than the 3+1-LOW fit. Moreover, the fact that both the global goodness-of-fit and the appearance-disappearance parameter goodness-of-fit are acceptable in the 3+1-HIG fit tells us that the fit is reliable. Hence, in Figs. 2 and 3 we present the allowed regions in the $\sin ^{2} 2 \vartheta_{e \mu}-\Delta m_{41}^{2}$, $\sin ^{2} 2 \vartheta_{e e}-\Delta m_{41}^{2}$ and $\sin ^{2} 2 \vartheta_{\mu \mu}-\Delta m_{41}^{2}$ planes, which are relevant, respectively, for $\left.\stackrel{(-)}{\nu_{\mu}} \rightarrow \stackrel{(-)}{\nu}\right)$ appearance, $\stackrel{(-)}{\nu}$ dis-

3 The interesting possibility of reconciling the low-energy anomalous data with neutrino oscillations through energy reconstruction effects proposed in [62, 63] still needs a detailed study. 

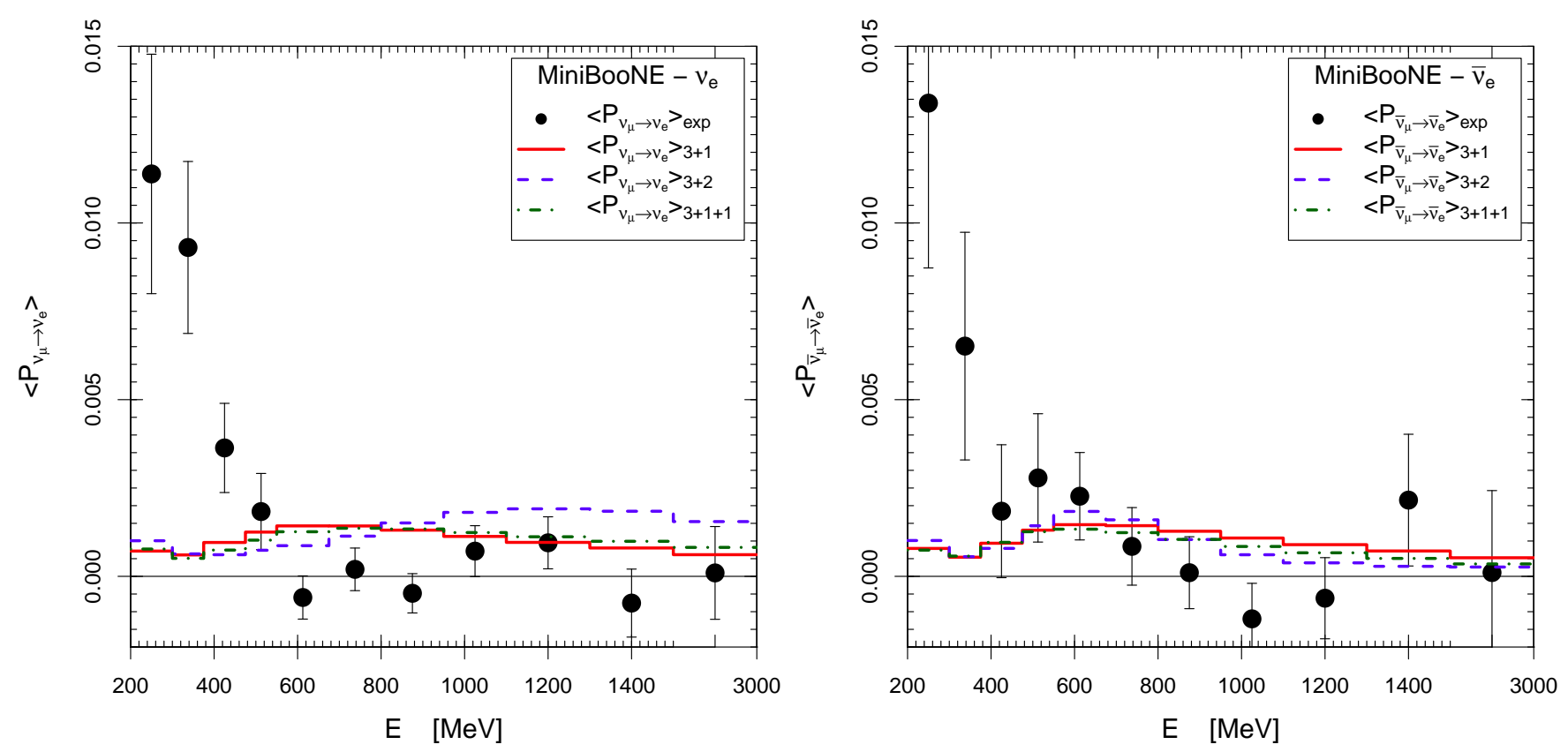

FIG. 1. Averaged transition probability in MiniBooNE bins corresponding to the best-fit values of the oscillation parameters in the $3+1-\mathrm{LOW}, 3+2-\mathrm{LOW}$ and 3+1+1-LOW fits (see Tabs. Iand II) compared with the experimental data.

appearance and $\stackrel{(-)}{\nu})$ disappearance searches. One can see that the allowed region is well defined, with

$$
0.82<\Delta m_{41}^{2}<2.19 \mathrm{eV}^{2} \quad(3 \sigma)
$$

Figure 2 shows also the region allowed by $\stackrel{(-)}{\nu_{\mu}} \rightarrow \stackrel{(-)}{\nu_{e}}$ appearance data and the constraints on $\sin ^{2} 2 \vartheta_{e \mu}$ from $\stackrel{(-)}{\nu}$ disappearance and $\stackrel{(-)}{\nu})$ disappearance data. One can see that the combined disappearance constraint excludes a large part of the region allowed by $\stackrel{(-)}{\nu}) \rightarrow \stackrel{(-)}{\nu}$ appearance data, leading to the well-known appearancedisappearance tension [31 36, 38, 39] quantified by the parameter goodness-of-fit in Tab. I] With respect to the results presented in Refs. 32 34, 36, 38], the region at $\Delta m_{41}^{2} \simeq 6 \mathrm{eV}^{2}$ which is allowed by $\stackrel{(-)}{\nu} \rightarrow \stackrel{(-)}{\nu}$ appearance data is not allowed any more (at $3 \sigma$ ) by the global fit, mainly because of the old BNL-E776 data, which we included following the wise approach of Ref. [39]. This is consistent with the cosmological exclusion of this region [36, 38].

It is interesting to investigate what is the impact of the MiniBooNE experiment towards the test of the LSND signal. With this aim we performed two additional $3+1$ fits: a 3+1-noMB fit without MiniBooNE data and a $3+1$-noLSND fit without LSND data. From Tab. I one can see that the results of the $3+1$-noMB fit are similar to those of the 3+1-HIG fit and the exclusion of the case of no-oscillations remains at the level of $6 \sigma$. On the other hand, in the 3+1-noLSND fit the exclusion of the case of

\begin{tabular}{ccccc}
\hline \hline & $3+2$ & $3+2$ & $3+1+1$ & $3+1+1$ \\
& LOW & HIG & LOW & HIG \\
\hline$\chi_{\min }^{2}$ & 284.4 & 256.4 & 289.8 & 259.0 \\
$\mathrm{NDF}$ & 252 & 246 & 253 & 247 \\
$\mathrm{GoF}$ & $8 \%$ & $31 \%$ & $6 \%$ & $29 \%$ \\
$\Delta m_{41}^{2}\left[\mathrm{eV}^{2}\right]$ & 1.9 & 0.93 & 1.6 & 1.6 \\
$\left|U_{e 4}\right|^{2}$ & 0.03 & 0.015 & 0.026 & 0.023 \\
$\left|U_{\mu 4}\right|^{2}$ & 0.012 & 0.0097 & 0.011 & 0.012 \\
$\Delta m_{51}^{2}\left[\mathrm{eV}^{2}\right]$ & 4.1 & 1.6 & & \\
$\left|U_{e 5}\right|^{2}$ & 0.013 & 0.018 & 0.0088 & 0.0092 \\
$\left|U_{\mu 5}\right|^{2}$ & 0.0065 & 0.0091 & 0.0049 & 0.0052 \\
$\eta / \pi$ & 0.51 & 1.6 & 0.4 & 0.45 \\
\hline$\left(\chi_{\min }^{2}\right)_{\mathrm{APP}}$ & 87.7 & 69.8 & 94.8 & 75.5 \\
$\left(\chi_{\min }^{2}\right)_{\mathrm{DIS}}$ & 179.1 & 179.1 & 180.1 & 180.1 \\
$\Delta \chi_{\mathrm{PG}}^{2}$ & 17.7 & 7.5 & 14.9 & 3.4 \\
$\mathrm{NDF}_{\mathrm{PG}}$ & 4 & 4 & 3 & 3 \\
$\mathrm{GoF}_{\mathrm{PG}}$ & $0.1 \%$ & $11 \%$ & $0.2 \%$ & $34 \%$ \\
\hline $\mathrm{p}-\mathrm{val}_{3+1}$ & 0.12 & 0.25 & 0.59 & 0.42 \\
$n \sigma_{3+1}$ & $1.6 \sigma$ & $1.2 \sigma$ & $0.54 \sigma$ & $0.8 \sigma$ \\
\hline \hline
\end{tabular}

TABLE II. Results of the fit of short-baseline data taking into account all MiniBooNE data (LOW) and only the MiniBooNE data above $475 \mathrm{MeV}$ (HIG) in the framework of $3+2$ and $3+1+1$ neutrino mixing. The notation is similar to that in Tab. I. The last two lines give the $\mathrm{p}$-value $\left(\mathrm{p}-\mathrm{val}_{3+1}\right)$ and the corresponding number of excluding $\sigma$ 's $\left(n \sigma_{3+1}\right)$ of the $3+1$ scheme.

no-oscillations drops dramatically te屯 $2.1 \sigma$. Therefore, it

4 This is due to the fact that without LSND the main indica- 


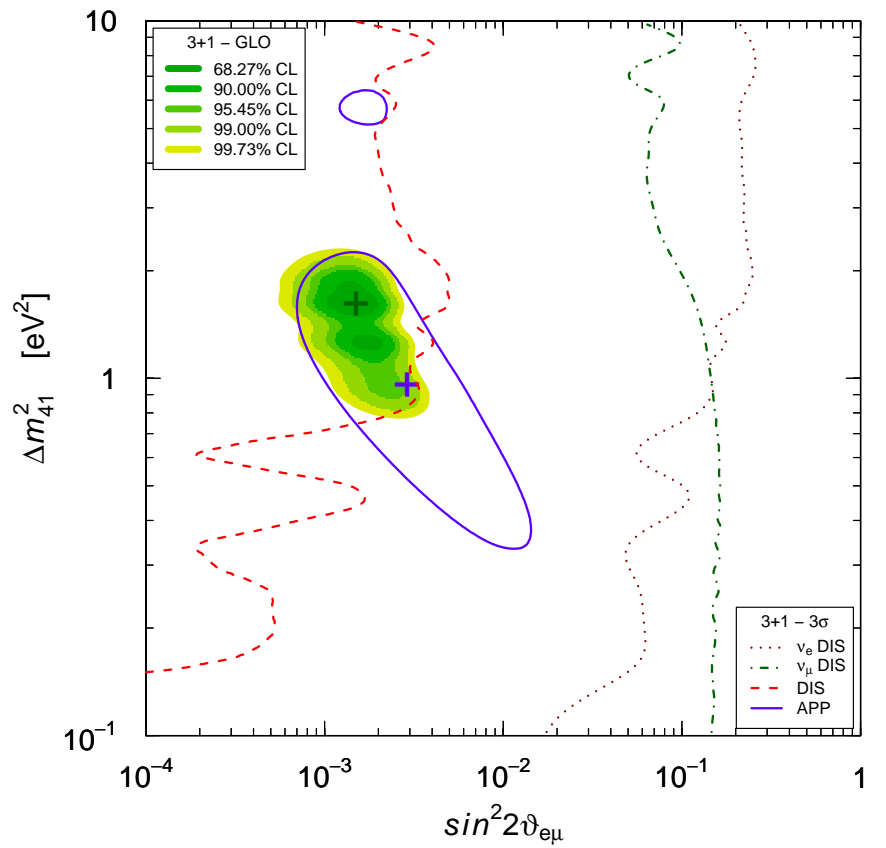

FIG. 2. Allowed region in the $\sin ^{2} 2 \vartheta_{e \mu}-\Delta m_{41}^{2}$ plane in the global (GLO) 3+1-HIG fit of short-baseline neutrino oscillation data compared with the $3 \sigma$ allowed regions obtained from $\stackrel{(-)}{\nu} \rightarrow \stackrel{(-)}{\nu}$ short-baseline appearance data (APP; inside the solid blue curves) and the $3 \sigma$ constraints obtained from $\stackrel{(-)}{\nu}$ short-baseline disappearance data $\left(\nu_{e}\right.$ DIS; left of the dotted dark-red curve), $\stackrel{(-)}{\nu_{\mu}}$ short-baseline disappearance data $\left(\nu_{\mu}\right.$ DIS; left of the dash-dotted dark-green curve) and the combined short-baseline disappearance data (DIS; left of the dashed red curve). The best-fit points of the GLO and APP fits are indicated by crosses.

is evident that the LSND experiment is still crucial for the indication in favor of short-baseline $\bar{\nu}_{\mu} \rightarrow \bar{\nu}_{e}$ transitions and the MiniBooNE experiment has been rather inconclusive.

Let us consider now the fit of short-baseline data in the framework of $3+2$ mixing, which was considered to be interesting in 2010 when the MiniBooNE neutrino 64 and antineutrino 65 data showed a CP-violating tension. Unfortunately, this tension reduced considerably in the final MiniBooNE data 20] and from Tab. II one can see that there is little improvement of the $3+2$ fit with respect to the $3+1$ case, in spite of the four additional parameters and the additional possibility of $\mathrm{CP}$

tion in favor of short-baseline oscillations is given by the reactor [46 48] and Gallium [53] anomalies. In fact, the $2.1 \sigma$ exclusion $\left(\Delta \chi^{2} / \mathrm{NDF}=8.3 / 3\right)$ of the case of no-oscillations in the global fit of short-baseline neutrino oscillation data without LSND is comparable with the $2.7 \sigma$ exclusion $\left(\Delta \chi^{2} / \mathrm{NDF}=10.1 / 2\right)$ that we obtain from the analysis of $\left(\bar{\nu}_{e}\right)$ short-baseline disappearance data alone [18].

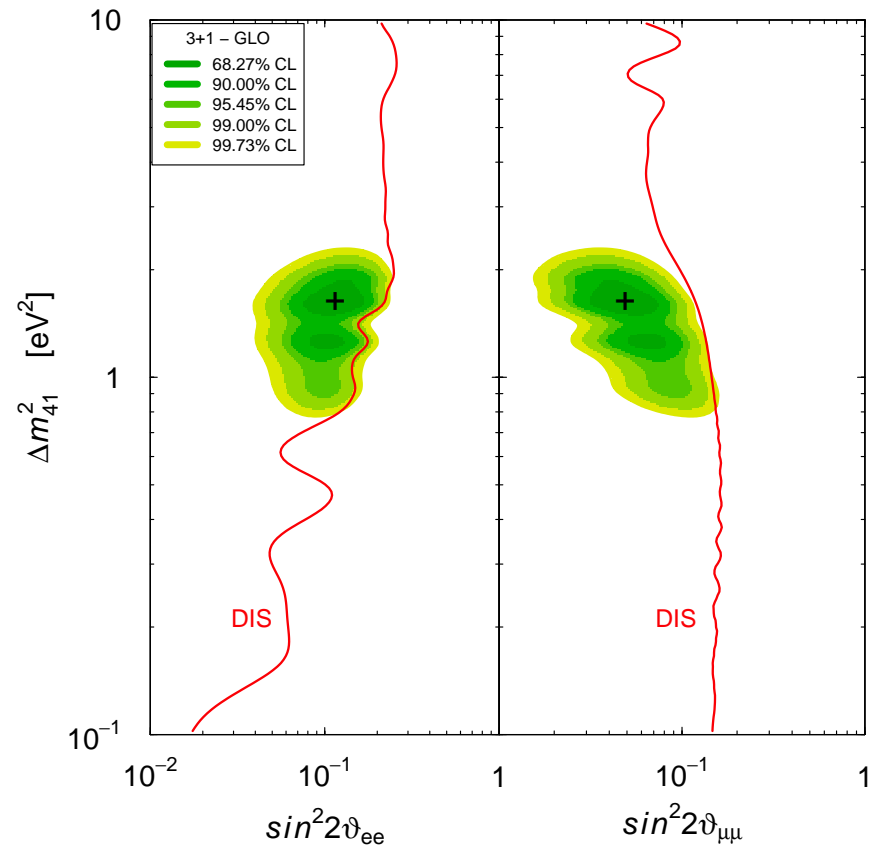

FIG. 3. Allowed regions in the $\sin ^{2} 2 \vartheta_{e e^{-\Delta}} m_{41}^{2}$ and $\sin ^{2} 2 \vartheta_{\mu \mu}-\Delta m_{41}^{2}$ planes in the global (GLO) $3+1-$ HIG fit of short-baseline neutrino oscillation data compared with the $3 \sigma$ constraints obtained from $\left(\bar{\nu}_{e}\right)$ short-baseline disappearance data (solid red DIS curve in the left panel),,$\stackrel{(-)}{\nu}$ short-baseline disappearance data (solid red DIS curve in the right panel). The best-fit point of the GLO fit is indicated by crosses.

violation. First, from Fig. 1 one can see that the $3+2-$ LOW fit is as bad as the $3+1-\mathrm{LOW}$ fit in fitting the three anomalous MiniBooNE low-energy bins5. Moreover, comparing Tabs. [1 and II one can see that the appearance-disappearance tension in the $3+2$-LOW fit is even worse than that in the $3+1-\mathrm{LOW}$ fit, since the $\Delta \chi_{\mathrm{PG}}^{2}$ is so much larger that it cannot be compensated by the additional degrees of freedom (this behavior has been explained in Ref. 38]). Hence, as in the $3+1$ case it is wise to neglect the three low-energy MiniBooNE anomalous bins and consider as more reliable the $3+2$-HIG fit, which has an acceptable appearance-disappearance parameter goodness-of-fit. However, one must ask if considering the larger complexity of the $3+2$ scheme is justified by the data. The answer is negativ 6 because, as one can see from Tab. III, the value of the p-value obtained by restricting the $3+2$ scheme to $3+1$ disfavors the $3+1$ scheme only at $1.2 \sigma$ in the $3+2$-HIG fit.

5 One could fit the three anomalous MiniBooNE low-energy bins in a $3+2$ scheme 35] by considering the appearance data without the ICARUS [44] and OPERA [45] constraints, but the corresponding relatively large transition probabilities are excluded by the disappearance data.

6 See however the somewhat different conclusions reached in Ref. 39]. 
A puzzling feature of the $3+2$ scheme is that it needs the existence of two sterile neutrinos with masses at the eV scale. We think that it may be considered as more plausible that sterile neutrinos have a hierarchy of masses. Hence, we considered also the $3+1+1$ scheme 66 69] in which $m_{5}$ is much heavier than $1 \mathrm{eV}$ and the oscillations due to $\Delta m_{51}^{2}$ are averaged. Hence, in the analysis of short-baseline data the $3+1+1$ scheme has one effective parameter less than the $3+2$ scheme. The results of the $3+1+1$-LOW and $3+1+1-$ HIG fits presented in Tab. II show that the $3+1+1-\mathrm{LOW}$ is as bad as the $3+1$-LOW and $3+2$-LOW fits (see also the bad fit of the three low-energy MiniBooNE anomalous bins in Fig. 11). On the other hand, the $3+1+1-$ HIG appearancedisappearance parameter goodness-of-fit is remarkably good, with a $\Delta \chi_{\mathrm{PG}}^{2}$ that is smaller than those in the $3+1-\mathrm{HIG}$ and $3+2-\mathrm{HIG}$ fits. However, the $\chi_{\min }^{2}$ in the $3+1+1$-HIG is only slightly smaller than that in the $3+1$ HIG fit and the high p-value of the $3+1$ scheme does not allow us to prefer the more complex $3+1+1$.

In conclusion, we have presented the results of the global analysis of all the available data of short-baseline neutrino oscillation experiments in the framework of $3+1$, $3+2$ and $3+1+1$ neutrino mixing schemes. We have shown that the data do not allow us to reject the simplest $3+1$ scheme in favor of the more complex $3+2$ and
$3+1+1$ schemes. We have also shown that the low-energy MiniBooNE anomaly cannot be explained by neutrino oscillations in any of these schemes. Considering the preferred $3+1$ scheme, we have updated the constraints on the oscillation parameters and we have shown that there is only one allowed region in the parameter space around $\Delta m_{41}^{2} \simeq 1-2 \mathrm{eV}^{2}$. We have also shown that the crucial indication in favor of short-baseline $\bar{\nu}_{\mu} \rightarrow \bar{\nu}_{e}$ appearance is still given by the old LSND data and the MiniBooNE experiment has been inconclusive. Hence new better experiments are needed in order to check this signal [2, 70 72]. Let us finally emphasize that, besides the direct observation of short-baseline $\stackrel{(-)}{\nu}_{\mu} \rightarrow \stackrel{(-)}{\nu}$ transitions, it is crucial to observe also short-baseline $\stackrel{(-)}{\nu_{e}}$ and $\stackrel{(-)}{\nu_{\mu}}$ disappearance. Since the reactor and Gallium anomalies indicate that ${ }^{(-)} \nu_{e}$ indeed disappear, it is important to search also for the disappearance of $\stackrel{(-)}{\nu}_{\mu}[73,74]$.

\section{ACKNOWLEDGMENT}

The work of H.W.L. is supported in part by the National Natural Science Foundation of China under Grant No. 11265006. The work of Y.F.L. is supported in part by the National Natural Science Foundation of China under Grants No. 11135009 and No. 11305193.
[1] M. C. Gonzalez-Garcia and M. Maltoni, Phys. Rept. 460, 1 (2008), arXiv:0704.1800

[2] K. N. Abazajian et al., arXiv:1204.5379.

[3] A. Palazzo, Mod.Phys.Lett. A28, 1330004 (2013), arXiv:1302.1102.

[4] M. Drewes, International Journal of Modern Physics E, Vol. 22, 1330019 (2013), arXiv:1303.6912

[5] R. R. Volkas, Prog. Part. Nucl. Phys. 48, 161 (2002), hep-ph/0111326.

[6] R. N. Mohapatra and A. Y. Smirnov, Ann. Rev. Nucl. Part. Sci. 56, 569 (2006), hep-ph/0603118

[7] A. Diaferio and G. W. Angus, arXiv:1206.6231.

[8] S. Riemer-Sorensen, D. Parkinson, and T. M. Davis, arXiv:1301.7102,

[9] M. Archidiacono, E. Giusarma, S. Hannestad, and O. Mena, arXiv:1307.0637

[10] Planck, P. A. R. Ade et al., arXiv:1303.5076.

[11] A. Mirizzi et al., Phys. Lett. B726, 8 (2013), arXiv:1303.5368.

[12] P. Di Bari, S. F. King, and A. Merle, Phys.Lett. B724, 77 (2013), arXiv:1303.6267.

[13] E. D. Valentino, A. Melchiorri, and O. Mena, arXiv:1304.5981.

[14] N. Said, E. D. Valentino, and M. Gerbino, Phys.Rev. D88, 023513 (2013), arXiv:1304.6217.

[15] L. Verde, S. M. Feeney, D. J. Mortlock, and H. V. Peiris, JCAP 1309, 013 (2013), arXiv:1307.2904.

[16] M. Wyman, D. H. Rudd, R. A. Vanderveld, and W. Hu, arXiv:1307.7715.
[17] J. Hamann and J. Hasenkamp, JCAP 1310, 044 (2013), arXiv:1308.3255

[18] C. Giunti, M. Laveder, Y. Li, Q. Liu, and H. Long, Phys. Rev. D86, 113014 (2012), arXiv:1210.5715.

[19] LSND, A. Aguilar et al., Phys. Rev. D64, 112007 (2001), hep-ex/0104049.

[20] MiniBooNE, A. Aguilar-Arevalo et al., Phys.Rev.Lett. 110, 161801 (2013), arXiv:1303.2588

[21] A. Boyarsky, O. Ruchayskiy, and M. Shaposhnikov, Ann. Rev. Nucl. Part. Sci. 59, 191 (2009), arXiv:0901.0011.

[22] A. Kusenko, Phys. Rept. 481, 1 (2009), arXiv:0906.2968.

[23] S. Gardner and G. Fuller, Prog.Part.Nucl.Phys. 71, 167 (2013), arXiv:1303.4758.

[24] A. Boyarsky, D. Iakubovskyi, and O. Ruchayskiy, Phys.Dark Univ. 1, 136 (2012), arXiv:1306.4954

[25] C. Das, J. Pulido, and M. Picariello, Phys. Rev. D79, 073010 (2009), arXiv:0902.1310

[26] P. C. de Holanda and A. Y. Smirnov, Phys. Rev. D83, 113011 (2011), arXiv:1012.5627

[27] A. de Gouvea and T. Wytock, Phys. Rev. D79, 073005 (2009), arXiv:0809.5076.

[28] P. Bakhti and Y. Farzan, arXiv:1308.2823

[29] A. Palazzo, JHEP 1310, 172 (2013), arXiv:1308.5880.

[30] A. Esmaili, E. Kemp, O. L. G. Peres, and Z. Tabrizi, Phys.Rev. D88, 073012 (2013), arXiv:1308.6218.

[31] J. Kopp, M. Maltoni, and T. Schwetz, Phys. Rev. Lett. 107, 091801 (2011), arXiv:1103.4570

[32] C. Giunti and M. Laveder, Phys.Rev. D84, 073008 (2011), arXiv:1107.1452. 
[33] C. Giunti and M. Laveder, Phys.Rev. D84, 093006 (2011), arXiv:1109.4033.

[34] C. Giunti and M. Laveder, Phys. Lett. B706, 200 (2011), arXiv:1111.1069.

[35] J. Conrad, C. Ignarra, G. Karagiorgi, M. Shaevitz, and J. Spitz, Adv.High Energy Phys. 2013, 163897 (2013), arXiv:1207.4765.

[36] M. Archidiacono, N. Fornengo, C. Giunti, and A. Melchiorri, Phys. Rev. D86, 065028 (2012), arXiv:1207.6515.

[37] C. Giunti, M. Laveder, Y. Li, and H. Long, Phys. Rev. D87, 013004 (2013), arXiv:1212.3805.

[38] M. Archidiacono, N. Fornengo, C. Giunti, S. Hannestad, and A. Melchiorri, Phys.Rev. D87, 125034 (2013), arXiv:1302.6720.

[39] J. Kopp, P. A. N. Machado, M. Maltoni, and T. Schwetz, JHEP 1305, 050 (2013), arXiv:1303.3011.

[40] C. Giunti and C. W. Kim, Fundamentals of Neutrino Physics and Astrophysics (Oxford University Press, Oxford, UK, 2007), ISBN 978-0-19-850871-7.

[41] BNL-E776, L. Borodovsky et al., Phys. Rev. Lett. 68, 274 (1992).

[42] KARMEN, B. Armbruster et al., Phys. Rev. D65, 112001 (2002), hep-ex/0203021.

[43] NOMAD, P. Astier et al., Phys. Lett. B570, 19 (2003), hep-ex/0306037.

[44] ICARUS, M. Antonello et al., arXiv:1307.4699.

[45] OPERA, N. Agafonova et al., JHEP 1307, 004 (2013), arXiv:1303.3953.

[46] T. A. Mueller et al., Phys. Rev. C83, 054615 (2011), arXiv:1101.2663.

[47] G. Mention et al., Phys. Rev. D83, 073006 (2011), arXiv:1101.2755.

[48] P. Huber, Phys. Rev. C84, 024617 (2011), arXiv:1106.0687.

[49] SAGE, J. N. Abdurashitov et al., Phys. Rev. C73, 045805 (2006), nucl-ex/0512041

[50] M. Laveder, Nucl. Phys. Proc. Suppl. 168, 344 (2007), Workshop on Neutrino Oscillation Physics (NOW 2006), Otranto, Lecce, Italy, 9-16 Sep 2006.

[51] C. Giunti and M. Laveder, Mod. Phys. Lett. A22, 2499 (2007), hep-ph/0610352
[52] M. A. Acero, C. Giunti, and M. Laveder, Phys. Rev. D78, 073009 (2008), arXiv:0711.4222.

[53] C. Giunti and M. Laveder, Phys. Rev. C83, 065504 (2011), arXiv:1006.3244

[54] CDHSW, F. Dydak et al., Phys. Lett. B134, 281 (1984).

[55] M. Maltoni and T. Schwetz, Phys. Rev. D76, 093005 (2007), arXiv:0705.0107

[56] A. Esmaili, F. Halzen, and O. L. G. Peres, JCAP 1211, 041 (2012), arXiv:1206.6903

[57] A. Esmaili and A. Y. Smirnov, arXiv:1307.6824.

[58] MINOS, P. Adamson et al., Phys. Rev. Lett. 107, 011802 (2011), arXiv:1104.3922

[59] SciBooNE-MiniBooNE, K. B. M. Mahn et al., Phys. Rev. D85, 032007 (2012), arXiv:1106.5685.

[60] SciBooNE-MiniBooNE, G. Cheng et al., Phys. Rev. D86, 052009 (2012), arXiv:1208.0322

[61] M. Maltoni and T. Schwetz, Phys. Rev. D68, 033020 (2003), hep-ph/0304176

[62] M. Martini, M. Ericson, and G. Chanfray, Phys. Rev. D85, 093012 (2012), arXiv:1202.4745.

[63] M. Martini, M. Ericson, and G. Chanfray, Phys. Rev. D87, 013009 (2013), arXiv:1211.1523.

[64] MiniBooNE, A. A. Aguilar-Arevalo et al., Phys. Rev. Lett. 102, 101802 (2009), arXiv:0812.2243.

[65] MiniBooNE, A. A. Aguilar-Arevalo et al., Phys. Rev. Lett. 105, 181801 (2010), arXiv:1007.1150.

[66] A. E. Nelson, Phys. Rev. D84, 053001 (2011), arXiv:1010.3970

[67] J. Fan and P. Langacker, JHEP 04, 083 (2012), arXiv:1201.6662

[68] E. Kuflik, S. D. McDermott, and K. M. Zurek, Phys. Rev. D86, 033015 (2012), arXiv:1205.1791

[69] J. Huang and A. E. Nelson, Phys.Rev. D88, 033016 (2013), arXiv:1306.6079.

[70] C. Rubbia, A. Guglielmi, F. Pietropaolo, and P. Sala, arXiv:1304.2047

[71] OscSNS, M. Elnimr et al., arXiv:1307.7097.

[72] J.-P. Delahaye et al., arXiv:1308.0494.

[73] D. Adey et al., arXiv:1305.1419.

[74] L. Stanco, S. Dusini, A. Longhin, A. Bertolin, and M. Laveder, arXiv:1306.3455. 